\title{
Altered callose deposition during embryo sac formation of multi-pistil mutant (mp1) in Medicago sativa
}

\author{
H.C. Zhou ${ }^{1}$, L. Jin ${ }^{2}$, J. Li ${ }^{1}$ and X.J. Wang ${ }^{2}$ \\ ${ }^{1}$ School of Pastoral Agriculture Science and Technology, Lanzhou University, \\ Lanzhou, China \\ ${ }^{2}$ Natural History Research Center, Shanghai Natural History Museum, \\ Shanghai Science \& Technology Museum, Shanghai, China \\ Corresponding author: X.J. Wang \\ E-mail: wangxj@sstm.org.cn
}

Genet. Mol. Res. 15 (2): gmr.15027698

Received October 30, 2015

Accepted February 12, 2016

Published June 3, 2016

DOI http://dx.doi.org/10.4238/gmr.15027968

\begin{abstract}
Whether callose deposition is the cause or result of ovule sterility in Medicago sativa remains controversial, because it is unclear when and where changes in callose deposition and dissolution occur during fertile and sterile embryo sac formation. Here, alfalfa spontaneous multi-pistil mutant ( $\mathrm{mpl}$ ) and wild-type plants were used to compare the dynamics of callose deposition during embryo sac formation using microscopy. The results showed that both mutant and wild-type plants experienced megasporogenesis and megagametogenesis, and there was no significant difference during megasporogenesis. In contrast to the wild-type plants, in which the mature embryo sac was observed after three continuous cycles of mitosis, functional megaspores of mutant plants developed abnormally after the second round of mitosis, leading to degeneration of synergid, central, and antipodal cells. Callose deposition in both mutant and wild-type plants was first observed in the walls of megasporocytes, and then in the megaspore tetrad walls. After meiosis, the callose wall began to degrade as the functional
\end{abstract}


megaspore underwent mitosis, and almost no callose was observed in the mature embryo sac in wild-type plants. However, callose deposition was observed in $\mathrm{mpl}$ plants around the synergid, and increased with the development of the embryo sac, and was mainly deposited at the micropylar end. Our results indicate that synergid, central, and antipodal cells, which are surrounded by callose, may degrade owing to lack of nutrition. Callose accumulation around the synergid and at the micropylar end may hinder signals required for the pollen tube to enter the embryo sac, leading to abortion.

Key words: Medicago sativa; Multi-pistil mutant; Embryo sac; Callose; Abortion

\section{INTRODUCTION}

Alfalfa (Medicago sativa) is an autotetraploid cross-pollination crop and a perennial forage legume, which has the longest cultivation history and the widest distribution in the world. It is known as the "Queen of the Forages" owing to its high yield, good quality, fine palatability, and high economic value (Pasumarty et al., 1993; Bhandari et al., 2007). High seed yield has been a goal of recent alfalfa-breeding programs, and there has been an increasing demand for high-quality forage grass owing to the expanding livestock population and feed shortage in some areas. However, the actual seed yield of alfalfa is much lower than its potential seed yield, which is associated with the high percentage of female sterility (Lorenzetti, 1993). Female sterility is a common phenomenon in flowering plants, especially in perennial cross-pollination legumes (Teng et al., 2006; Nair et al., 2008; Jin et al., 2014). Pistal mutants account for some female sterility. Female organ mutants, such as pistillode, variation of pistil number, and mutation of pistil length, have been reported for wheat (Triticum aestivum) (Yang et al., 2011), rice (Oryza sativa) (Li et al., 2006), soybean (Glycine max) (Palmer and Horner, 2000; Palmer et al., 2008), pearl millet (Pennisetum glaucum) (Arthur et al., 1993), ramie (Boehmeria nivea), and rape (Brassica campestris) (Fu et al., 2014). The spontaneous multi-pistil mutant $m p 1$ was screened and identified in a natural alfalfa population in our previous breeding program, and was found to exhibit 2-3 pistils compared with the wild-type plant. Pod-setting of $m p 1$ plants was significantly lower $(8.18 \%)$ than that of wild-type plants $(71.43 \%)$, which indicated that $m p 1$ was partial-female-sterile. Furthermore, $m p 1$ is a new floral homeotic mutant with partial female sterility due to abnormal female gametogenesis, stigma development, and pollen tube guidance (Jin et al., 2014). Several studies have found ovule sterility to be related to callose deposition in Medicago species (Barcaccia et al., 1996; Wang et al., 2011). However, whether callose deposition is the cause or the result of ovule sterility in legume plants remains controversial.

Callose, a polysaccharide composed of $\beta$-1,3-glucan, is involved in various biological processes in plants, and is a component of specialized cell walls or cell wall-associated structures at particular stages of growth and differentiation (Tao et al., 2012; Liu et al., 2015). Callose may play a vital role during reproductive processes occurring in the anthers and ovules of flowering plants, and functions as a molecular filter between genetically different cells. In addition, it serves as a selective barrier to signals that are essential for meiosis (Barcaccia et 
al., 1996; Musiał et al., 2015). Moreover, callose deposition is vital during sexual reproduction in plants because it separates developing pollen grains, thus preventing their exine from fusing (Nishikawa et al., 2005; Li et al., 2011). Samuel et al. (2009) found that disorders of callose metabolism could affect ovule sterility in Arabidopsis. De Martinis and Mariani (1999) reported that although transgenic tobacco (Nicotiana tabacum) could be pollinated, pollen tube guidance into the micropylar end was abnormal, with high levels of callose deposition occurring during ovule development. In addition, callose deposition was once considered the result of plant male sterility rather than the cause, because its synthesis and degradation are closely associated with the development of tapetum tissue (Bione et al., 2002). However, in female-sterile plants, callose has been predominantly examined in cell walls or at specific positions of the ovules, and the dynamics of callose deposition during fertile and sterile embryo sac formation have not be described. Therefore, it remains unclear when and where callose deposition and dissolution occur during embryo sac development, and whether callose deposition is the cause or result of ovule sterility in female-sterile plants, especially in alfalfa.

Therefore, spontaneous multi-pistil mutant $\mathrm{mpl}$ plants may be valuable for exploring the role of callose in plant sexual reproduction. Our goal was to elucidate the molecular mechanisms leading to female sterility and low seed yield in M. sativa; therefore, the study of embryo sac development in female-sterile alfalfa was essential. To the best of our knowledge, fewer callose events during gametophyte development have been studied in the alfalfa multi-pistil mutant. In this study, the characteristics of callose metabolism during embryo sac development and in the alfalfa spontaneous multi-pistil mutant $m p 1$ were investigated to elucidate the relationship between the dynamics of callose metabolism and female sterility.

\section{MATERIAL AND METHODS}

\section{Plant materials}

Alfalfa spontaneous multi-pistil mutant $m p l$ and fertile plants (denoted wild-type) from the same line were derived from a large-scale screening of sterile mutants in a national alfalfa-breeding program. The multi-pistil mutant $\mathrm{mpl}$ belongs to a partial-female-sterile plant containing 2-3 pistils, with low seed-setting, and a percentage of multi-pistil florets per raceme that varies from 45.8 to $70.3 \%$ (Jin et al., 2014). Clonally propagated $\mathrm{mpl}$ and wild-type plants were obtained by stem cuttings and maintained in a greenhouse. The average greenhouse temperatures during the experiment were $25^{\circ} \pm 2^{\circ} \mathrm{C}$ in day $(16 \mathrm{~h})$ and $18^{\circ} \pm 2^{\circ} \mathrm{C}$ at night $(8 \mathrm{~h})$. The photosynthetic photon flux density was $>600 \mu \mathrm{mol} \cdot \mathrm{m}^{-2} \cdot \mathrm{s}^{-1}$. The relative humidity is 50-60\%. In 2013, 50 mutant $m p 1$ and wild-type clonal plants were transplanted at the Linze Experimental Station $\left(39^{\circ} 21^{\prime} \mathrm{N}, 100^{\circ} 07^{\prime} \mathrm{E}\right.$, altitude $\left.1367 \mathrm{~m}\right)$ in the Hexi Corridor, northwest China. At this site, alfalfa flower from mid-May to late July (Wang et al., 2009). The floret development period was divided into eight stages according to the method described by Wang et al. (2011) (Table 1). Florets were sampled at anthesis three times per year, in two successive years, from 2013 to 2014. For each stage, 40 florets of $m p 1$ and wild-type plants were randomly sampled. Florets and pods were photographed using a Nikon 4500 digital camera (Nikon Corporation, Tokyo). Images were processed using the Photoshop CS3 software (Adobe Systems, 2007). 
Table 1. Morphological characteristics of eight developmental stages of alfalfa flower.

\begin{tabular}{l|c|c|l}
\hline Stage & Floret length $(\mathrm{mm})$ & Pistil length $(\mathrm{mm})$ & Characteristics \\
\hline 1 & 2.7 & 1.9 & Flower bud green and wrapped; calyx light green and transparent \\
\hline 2 & 3.1 & 2.4 & Flower bud base inflated, green of calyx darkened \\
\hline 3 & 4.6 & 3.2 & Flower bud enlarged, color of calyx changed from light to dark \\
\hline 4 & 5.5 & 4.6 & Flower bud base became smaller, pistil developed \\
\hline 5 & 6.0 & 5.2 & Light purple petals out of the calyx \\
\hline 6 & 6.5 & 5.7 & Calyx turned yellow, calyx wrapped 3/4 flower bud, purple appeared in the petals \\
\hline 7 & 7.6 & 6.6 & Calyx wrapped 1/2 flower bud, obvious purple petals, mature pollen \\
\hline 8 & 8.4 & 7.5 & Valgus keel petal, fully mature floret \\
\hline
\end{tabular}

\section{Light microscopy}

Flower buds from eight different developmental stages of wild-type and $m p 1$ plants were collected (Figure 1A). Florets were fixed in formaldehyde, acetic acid and ethanol (90 $\mathrm{mL} \mathrm{70 \%} \mathrm{ethanol,} 5 \mathrm{~mL}$ formalin, $5 \mathrm{~mL}$ acetic acid) for $18-24 \mathrm{~h}$ and then stored in $70 \%$ ethanol until use. Pistils from wild-type plants (Figure 1B) and multi-pistillate in $m p 1$ plants (Figure 1D, E and F) were dissected from the florets. Samples were successively dehydrated in 50, 70,85 , and $95 \%$ ethanol for $2 \mathrm{~h}$, and then dehydrated in $100 \%$ ethanol for $1 \mathrm{~h}$. Next, samples were cleared in a mixed solution of anhydrous ethanol and xylene (3:1, 1:1, and 1:3), and then embedded in paraffin wax. Sections were cut to $4 \mu \mathrm{m}$ using an RM2135 rotary microtome (Leica Reichert, Heidelberger, Germany) and then stained with hematoxylin-eosin. All stained sections were observed under a light microscopy (Olympus-BX51, Olympus America Inc., Center Valley, PA, USA).

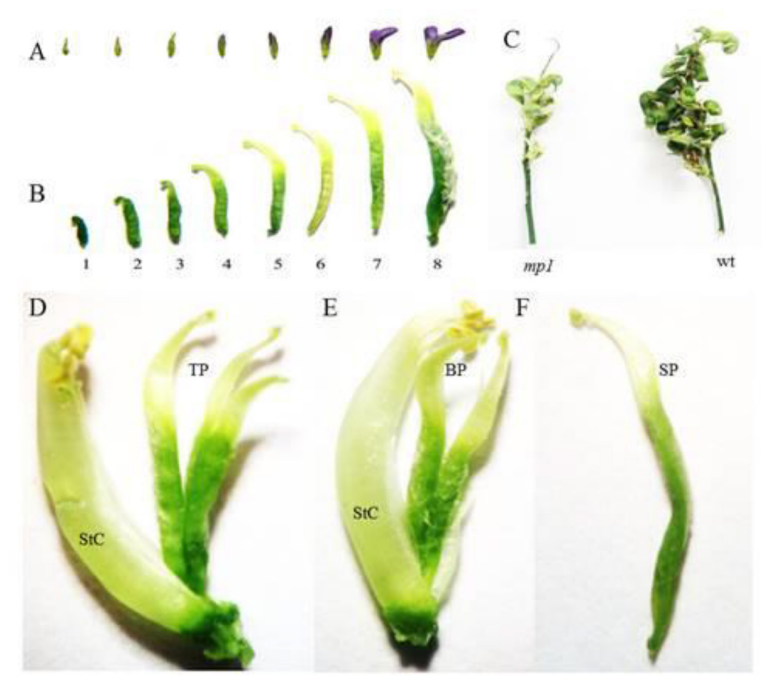

Figure 1. Floret and pistil of $m p 1$ and wild-type (wt) plants of Medicago sativa. A. and B. Floret and pistil at eight developmental stages (1-8). C. Seed-setting of $m p 1$ (left) and wild-type plants (right). D. $m p 1$ showing bi-pistil. E. $m p 1$ showing tri-pistil. F. Wild-type plant showing single-pistil. $\mathrm{SP}=$ single-pistil; $\mathrm{BP}=$ bi-pistil; $\mathrm{TP}=$ tri-pistil; $\mathrm{StC}=$ staminal column 


\section{Fluorescence microscopy}

Florets were fixed in Carnoy's solution (100\% ethanol:acetic acid $=3: 1)$ for a minimum of $24 \mathrm{~h}$ at room temperature. The pistils were then dissected, cleared in $8 \mathrm{M} \mathrm{NaOH}$ for $5 \mathrm{~h}$, washed twice under distilled water, and then stained overnight in $0.1 \%$ aniline blue in $0.1 \mathrm{M} \mathrm{K}_{3} \mathrm{PO}_{4}$ at $4^{\circ} \mathrm{C}$. Slides were overlaid with coverslips and sealed with 1-2 drops of aniline blue. Slides were generated for mutant and wild-type plants at each development stage and observed within $6 \mathrm{~h}$ to detect callose deposition using fluorescence microscopy (Olympus BX51, Olympus America Inc.).

To observe the dynamics of callose deposition during embryo sac development, florets from the different developmental stages of plant were sampled and fixed with Carnoy's solution for at least $24 \mathrm{~h}$. The pistils were dissected from florets and used to make paraffin sections as previously described. Several sections were then dewaxed in xylene for $5 \mathrm{~min}$, stained with $0.1 \%$ aniline blue for $2 \mathrm{~h}$ under dark conditions, and then observed under a fluorescence microscope (Olympus BX51, Olympus America Inc.) within 6 h.

\section{RESULTS}

\section{Embryo sac development in mp1 and wild-type $M$. sativa plants}

Pod-setting was significant lower in $m p 1$ plants (8.2\%) than in wild-type plants (71.4\%) (Figure 1C) (Jin et al., 2014). Analysis of female meiosis and embryo sac development in angiosperms is technically challenging, because the cells are enclosed within the nucellus and ovule tissues of the female flower. Using histology, embryo sac development of wild-type and mutant alfalfa (M. sativa) could be observed and divided into two phases. Phase 1 referred to megasporogenesis, during which archesporial cells developed and a functional megaspore formed. Phase 2 referred to megagametogenesis, during which the functional megaspore further developed into an embryo sac. Megasporogenesis does not differ between multi-pistillate florets and those of the wild-type plants. One or several archesporial cells, which were under the epidermis and were a larger size than surrounding cells (Figure 2A and I), underwent periclinal division to produce a primary sporogenous cell (Figure 2B and J). Then, the primary sporogenous cell directly developed into a megaspore mother cell (Figure $2 \mathrm{C}$ and $\mathrm{K}$ ), and the megaspore mother cell underwent two cycles of continuous meiosis, forming a tetrad that was arranged in a " $T$ " shape. Three megaspores near the micropylar end degenerated and the remaining one near the chalazal end developed into a functional megaspore (Figure 2D and L). In phase 2, the functional megaspore in wild-type plants developed into a mature embryo sac (seven cells, eight nuclear) after three cycles of normal mitosis (Figure 2E, F, G and H). Conversely, the functional megaspore of mutant plants developed abnormally at the beginning of the second round of mitosis. Nuclei at the micropylar end were observed to undergo normal mitosis, whereas antipodal cells at the chalazal end degraded (Figure $2 \mathrm{M}$ and $\mathrm{N}$ ), or the nuclei at the chalazal end developed normally, whereas the synergid at the micropylar end degraded (Figure 2O), resulting in ovule malformations (Figure 2P) and ultimately forming an abnormal embryo sac.

\section{Callose deposition in ovules of $m p 1$ and wild-type $M$. sativa plants}

Alfalfa wild-type plants showed a single-pistil trait with ovule curvature towards the 
base of the ovary at maturity (Figure 3A). Compared to the wild-type plant, the stylar canal region of $m p 1$ plants remained open, permitting the development of 2-3 successive pistils from a single-superior carpel primordium (Figure 3B and C). According to Rosellini et al. (1998) method, callose was visible as yellow-green fluorescence after aniline blue staining, with brighter fluorescence indicating higher callose deposition. Ovule development in alfalfa could be roughly classified into early and late developmental periods. In the early developmental period in wild-type and $m p l$ ovules, there was obvious callose deposition in the majority of nucellus (Figure 3D and F). No yellow-green fluorescence was observed with the development of ovule, that is, callose almost disappeared in the nucellus of wild-type plants as the ovule formed a mature embryo sac (Figure $3 \mathrm{G}$ ); however, callose was still present in the $\mathrm{mpl}$ nucellus (Figure 3E).
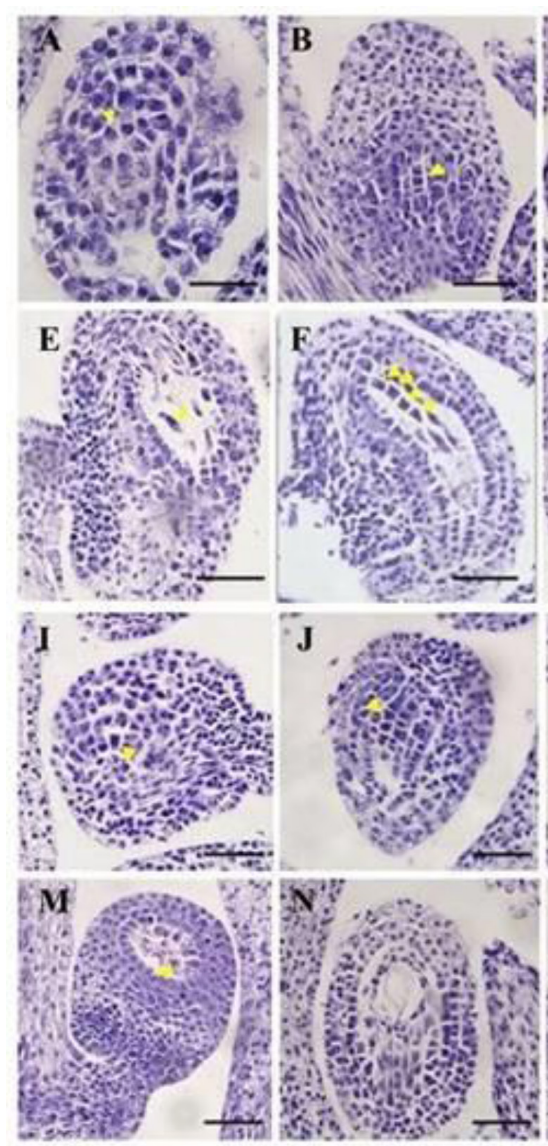

$\mathbf{J}$
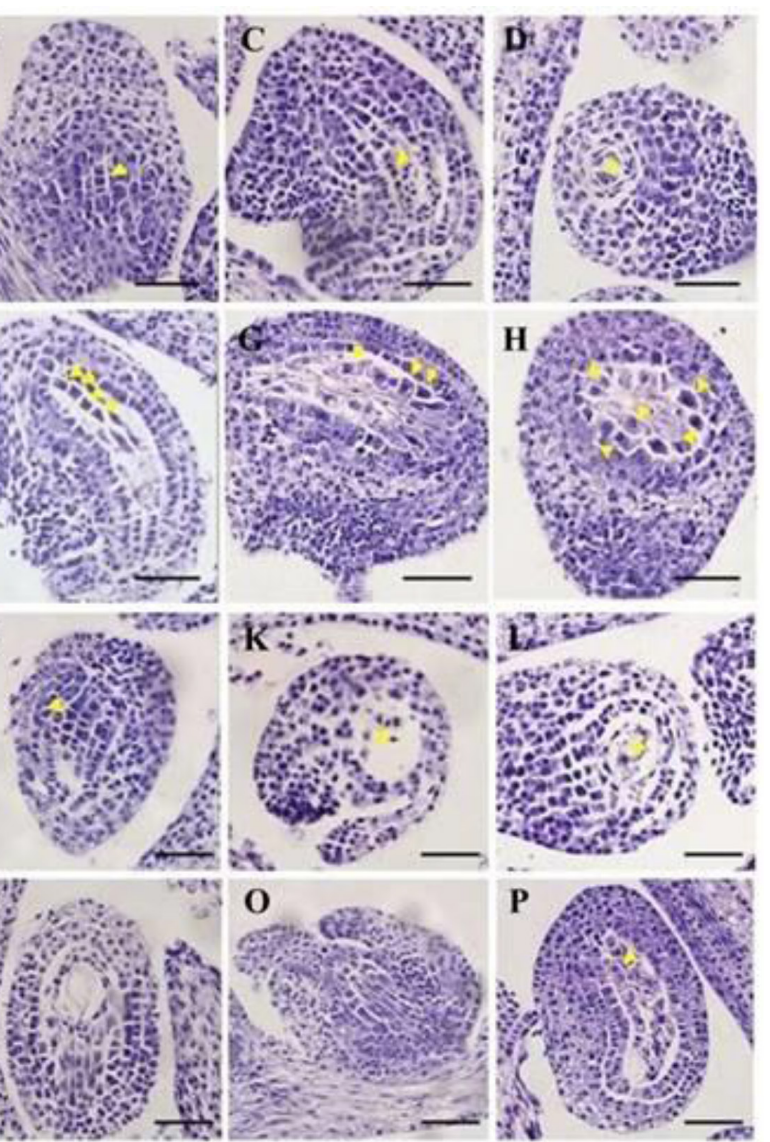

Figure 2. Embryo sac development in $m p l$ and wild-type plants of Medicago sativa. A.-H. Wild-type plant. I.-P. mp1. A. and I. Archesporial cell. B. and J. Archesporial cell undergoing a pericline division. C. and L. Megaspore mother cell. D. Functional megaspore away from the micropyle end. E. Functional megaspore undergoing the first mitosis. F. Functional megaspore undergoing the second mitosis. G. Functional megaspore undergoing the third mitosis. H. Mature embryo sac with seven cells and eight nuclei. K. Four megaspores distributing in a "T" shape. M. Functional megaspore undergoing mitosis. N. Abnormal nuclei at the chalazal end. O. Abnormal ovule development. P. Degenerated synergid at the micropylar end. Bar $=100 \mu \mathrm{m}$. 

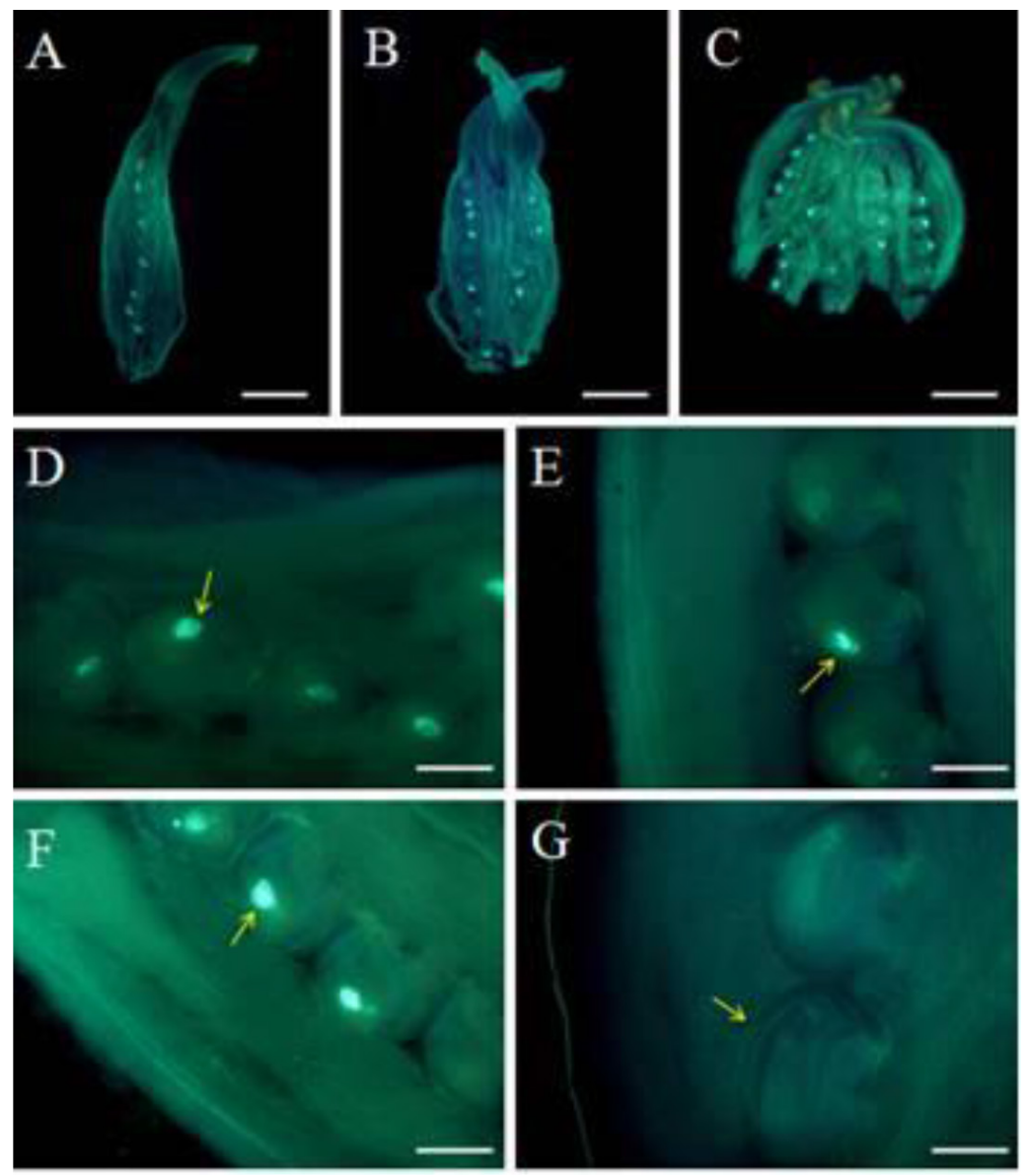

Figure 3. Callose deposition on ovules of wild-type and $\mathrm{mpl}$ plants of Medicago sativa. A. Single pistil of a wildtype plant. B. Double pistils of $m p 1$. C. Three pistils of $m p 1$. D. Visible callose deposition on nucellus during early development of $m p 1$ ovule. E. Callose deposition on nucellus during late development of the $m p 1$ ovule. F. Callose deposition on nucellus during early development of the wild-type ovule. G. Callose disappeared during the late stage of development of the wild-type ovule. Bar $=100 \mu \mathrm{m}(\mathrm{D}-\mathrm{G}) ; 200 \mu \mathrm{m}$ (A-C).

\section{Callose deposition dynamics in $m p 1$ and wild-type plants}

Fluorescence microscopy revealed almost no callose deposition in the course of periclinal division of archesporial cells and the production of primary sporogenous cells, which then formed megaspore mother cells (Figure 4A and I). Callose deposition in mutant and wild-type plants was first obvious in the walls of megaspore mother cells (Figure 4B and J). During meiosis, callose also accumulated in the walls of megaspore tetrads and in functional megaspores (Figure 4C, D, K and L). However, the callose wall began to degrade when the functional megaspore underwent its first mitosis (Figure 4E and M). The callose wall thinned, indicating that callose was being degraded throughout the process of embryo sac development (Figure 4E, F and G). Finally, it was difficult to detect callose deposition in the mature embryo 
sac of wild-type plants (Figure 4H). Conversely, after the functional megaspore completed its first mitosis, callose deposition in the $m p 1$ mutant occurred around the synergid and showed an increasing trend with the development of the embryo sac (Figure $4 \mathrm{~N}$ and $\mathrm{O}$ ), which was mainly deposited at the micropylar end. There was clear callose accumulation in the mature embryo sac of $m p l$ mutant plants (Figure 4P).
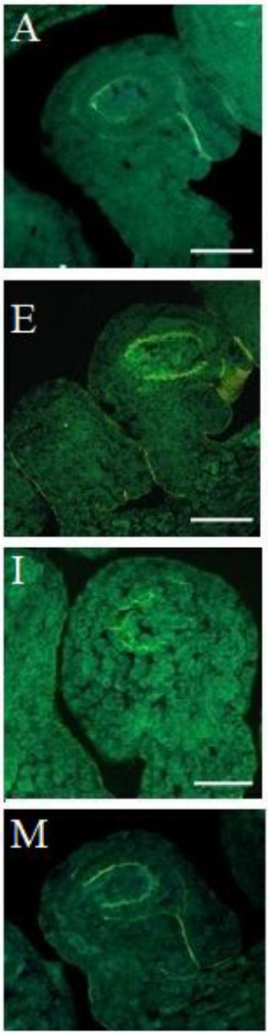
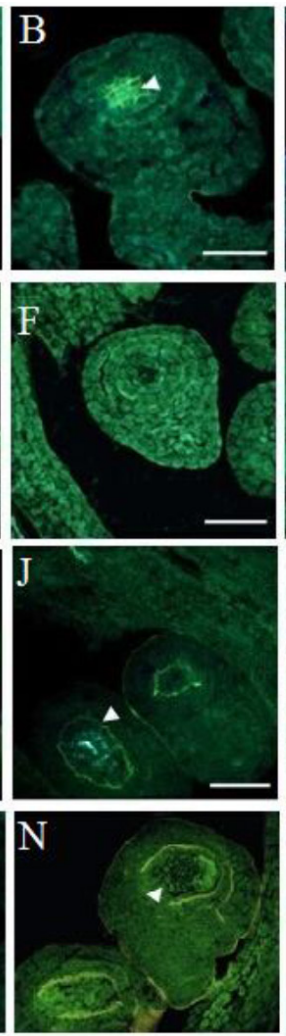
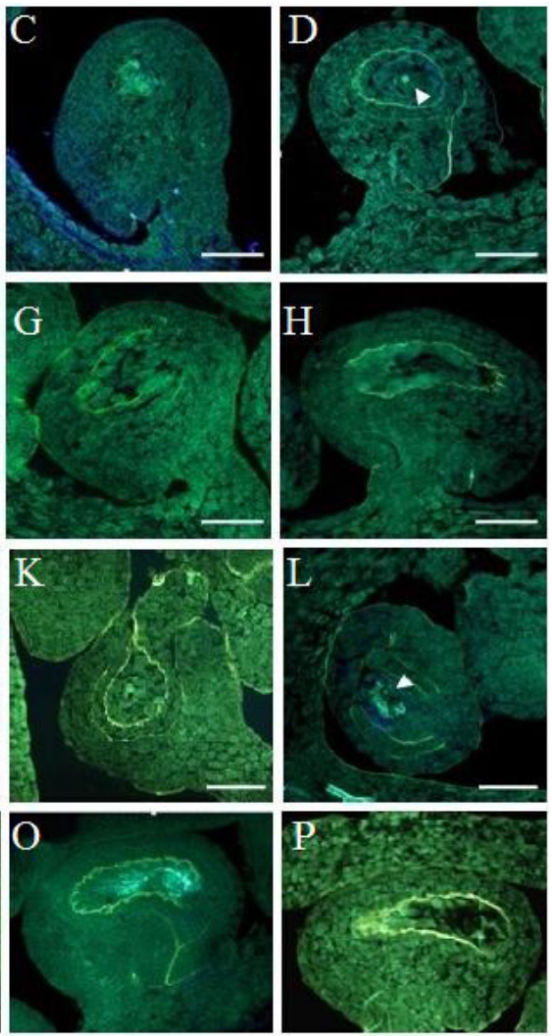

Figure 4. Callose deposition during embryo sac development in wild-type and $m p 1$ plants of Medicago sativa. A.-H. Wild-type plant. I.-P. $m p 1$. A. and I. Archesporial cell. B. and J. Megaspore mother cell. C. Megaspore mother cell forming tetrad after meiosis. D. Functional megaspore and callose wall. E.-G. Functional megaspore undergoing mitosis. H. Callose wall in a mature embryo sac. K. Megaspore mother cell forming a functional megaspore. L. Callose deposition around a functional megaspore. M.-O. During mitosis, callose reduced at an early stage and increased at a later stage. P. Part of a degraded embryo sac and callose deposited at the micropylar end. $\operatorname{Bar}=100 \mu \mathrm{m}$.

\section{DISCUSSION}

\section{Abnormal embryo sac development altered callose deposition in $m p 1$}

Normal development of the alfalfa embryo sac is the key to reproductive growth, and female gametophyte development is vital for its fertilization (Mariani et al., 1993; Ebel et al., 2004). Embryo sac development involves multiple cells undergoing differentiation in a serial 
and precisely controlled manner. This includes archesporial cell formation, megaspore mother cell differentiation, functional megaspore formation, and gamete cell differentiation (West and Harada, 1993). Any abnormal process could cause female sterility. Motamayor et al. (2000) reported a female-specific sterile mutant in A. thaliana, termed swil, which causes a delay in megasporogenes associated with an extra mitotic division of the megaspore mother cell in the majority of ovules. As a result, functional megaspore selection and gametogenesis are altered, and normal embryo sacs are not formed. In this study, embryo sac development was completely normal before functional megaspores underwent mitosis in $\mathrm{mpl}$ plants. However, after the first mitosis, $m p 1$ embryo sacs began to develop abnormally, mainly owing to synergid or antipodal cell degeneration, and embryo sac malformation. Developmental malformation of the synergid is detrimental to pollen tube growth, resulting in the pollen tube being unable to enter the embryo sac normally and ultimately leads to embryo abortion (Okuda et al., 2009). Moreover, in some extreme alfalfa sterile phenotypes, pistil development is limited in the abnormal embryo sac, and flower color is also affected in female-sterile plants (Rosellini et al., 2003). An efficient, easy to use Feulgen-based staining procedure has been described, which could be optimized for the identification of cell types in conjunction with confocal microscopy (Barrell and Grossniklaus, 2005).

Callose is thought to act as a temporary wall that prevents the products of meiosis from cohesion, fusion, and dissolution, which results in the release of free spores. Callose also functions as a molecular filter to isolate the developing meiocyte from the influence of external hormones and harmful substances (Tao et al., 2012; Winiarczyk et al., 2012). Other studies have shown that callose is usually deposited in the megaspore mother cell and in the meiosis tetrad during sexual reproduction (Rosellini et al., 2003; Musiał et al., 2015). The dynamic metabolism of callose in the walls of these cells may be related to cell fate and the switch from sporophyte development into megasporogenesis. In this study, callose was first deposited in the walls of megaspore mother cells and was degraded at the first mitosis. Callose gradually reduced over the process of embryo sac development and almost disappeared when the embryo sac matured in wild-type plants. This observation is consistent with the pattern of callose deposition during sexual reproduction in polygonumtype embryo sacs. In contrast, callose increased in $\mathrm{mpl}$ plants after mitosis of the functional megaspore. When the embryo sac matured, high levels of callose remained in the embryo sac, and it was deposited mainly in the micropylar end and in the synergid, consistent with previous findings (Rosellini et al., 1998). Callose deposited during megasporogenesis may act as a barrier to prevent interference from other cells, ensuring the formation of a functional megaspore.

However, in the present study, callose still accumulated during the late development of female-sterile ovule in alfalfa, and no callose deposition was observed in the wild-type plants. Vishnyakova (1991) studied the fluorescence of callose in mature ovules of seven species belonging to Leguminosae, Cruciferae, Solanaceae, and Gramineae, including alfalfa, red clover (Trifolium pratense), Caragana (Caragana arborescens), mustard (Brassica juncae), tomato (Lycopersicon esculentum), rye (Secale cereale), and barley (Hordeum vulgare). The results revealed no fluorescence in fertile ovules, whereas in the sterile ovule, different degrees of callose fluorescence were observed. Based on the above results, we could assess the sterility of plants through the deposition of callose in their mature ovules. The position and quantity of callose deposition, and fluorescence intensity in the sterile ovule are species dependent. 


\section{Changes in the dynamics of callose deposition predict female sterility}

Callose deposition around the megaspore is not conducive to embryo sac development, for example, the megasporogenesis of sickle alfalfa (M. falcata) and blue flower alfalfa (M. coerulea) are accompanied by callose deposition, which is thought to cause abnormal development of the embryo sac (Barcaccia et al., 1996). However, several studies have noted that the synthesis and degradation of callose is related to the development of tissue such as the tapetum (Zhang et al., 2007). Thus, callose deposition was once considered the result of plant sterility, rather than its cause (Bione et al., 2002). In this study, dynamics of callose deposition in wild-type and $m p 1$ plants were almost the same before functional megaspore underwent the first mitosis, i.e., high levels of callose were deposited from the time of the megaspore mother cell until the formation of a functional megaspore, and callose began to degrade at the first mitosis and then reduced to undetectable levels. However, when the functional megaspore has completed its first mitosis, callose is deposited around the synergid and its levels increase with the formation of mature embryo sac in $m p 1$ plants. This occurred very close to the time that the functional megaspore developed abnormally at the beginning of the second mitosis. Based on these results, it is speculated that abnormal callose deposition resulted in functional megaspore dysplasia, leading to embryo abortion. In addition, some research has shown that callose deposition and degradation change with microspore development, accounting for the observation that the dynamics of callose deposition are affected by embryo sac development (Ilarslan et al., 2003; Winiarczyk et al., 2012).

These results suggest that the disorder of callose metabolism causes functional megaspore to develop abnormally after the first mitosis. The continuous deposition of callose around the synergid could lead to its degradation owing to a lack of nutrition, and could also affect the expression of some genes, such as the overexpression of ZmEA1, which subsequently increases homologous protein concentration (Márton and Dresselhaus, 2008; Okuda et al., 2009). Callose may also hinder the secretion of chemical inducers from the synergid that could induce the growth of the pollen tube (Pagnussat et al., 2007), eventually resulting in female sterility in $m p 1$ plants. However, whether the ZmEA1-like gene is overexpressed or not remains to be determined. In alfalfa, female sterility due to recessive trait retarding integument development (Bingham and Hawkins, 1994), and quantitative ovule sterility associated with callose deposition (Rosellini et al., 1998) have been reported. Furthermore, gene expression analyses based on female sterile mutants should be performed. The identification of functional genes related to sac embryo development would be useful to clarify alfalfa female sterility in the future.

\section{Conflicts of interest}

The authors declare no conflict of interest.

\section{ACKNOWLEDGMENTS}

Research supported by the National Natural Science Foundation of China (\#31270558), and the Research Funds for the Introduction of Talents of Shanghai Science and Technology Museum. 


\section{REFERENCES}

Arthur L, Ozias-Akins P and Hanna WW (1993). Female sterile mutant in pearl millet: evidence for initiation of apospory. Heredity 84: 112-115.

Barcaccia G, Mazzucato A, Falcinelli M and Veronsei F (1996). Callose localization in cell walls during meiotic and apomeiotic megasporogenesis in diploid alfalfa (Medicago spp.). Caryologia 49: 45-56. http://dx.doi.org/10.1080/0 $\underline{0087114.1996 .10797349}$

Barrell PJ and Grossniklaus U (2005). Confocal microscopy of whole ovules for analysis of reproductive development: the elongate1 mutant affects meiosis II. Plant J. 43: 309-320. http://dx.doi.org/10.1111/j.1365-313X.2005.02456.x

Bhandari HS, Pierce CA, Murray LW and Ray IM (2007). Combining abilities and heterosis for forage yield among high-yielding accessions of the alfalfa core collection. Crop Sci. 47: 665-673. http://dx.doi.org/10.2135/ cropsci2006.06.0398

Bingham ET and Hawkins TW (1994). Female sterility in alfalfa due to a recessive trait retarding integument development. J. Hered. 75: 231-233.

Bione NCP, Pagliarini MS, Almeida LA and Seifert AL (2002). An $m s 2$ male sterile, female fertile soybean sharing phenotypic expression with other $m s$ mutants. Plant Breed. 121: 307-313. http://dx.doi.org/10.1046/j.14390523.2002.726806.x

De Martinis D and Mariani C (1999). Silencing gene expression of the ethylene-forming enzyme results in a reversible inhibition of ovule development in transgenic tobacco plants. Plant Cell 11: 1061-1072. http://dx.doi.org/10.1105/ tpc.11.6.1061

Ebel C, Mariconti L and Gruissem W (2004). Plant retinoblastoma homologues control nuclear proliferation in the female gametophyte. Nature 429: 776-780. http://dx.doi.org/10.1038/nature02637

Fu WQ, Zhao ZG, Ge XH, Ding L, et al. (2014). Anatomy and transcript profiling of gynoecium development in female sterile Brassica napus mediated by one alien chromosome from Orychophragmus violaceus. BMC Genomics 15: 61. http://dx.doi.org/10.1186/1471-2164-15-61

Ilarslan H, Horner HT and Palmer RG (2003). Megagametophyte abnormalities of near-isogenic female partial-sterile soybean mutants ( Glycine max; Leguminosae). J. Plant Res. 116: 141-149.

Jin L, Zhang JW, Li J, Yang Y, et al. (2014). Spontaneous multi-pistil mutant $m p 1$ in alfalfa: floral anatomy and embryo sac development. Agron. J. 106: 431-440. http://dx.doi.org/10.2134/agronj2013.0406

Li JJ, Liu L, Ouyang YD and Yao JL (2011). Sexual reproduction development in apomictic Eulaliopsis binata (Poaceae). Genet. Mol. Res. 10: 2326-2339. http://dx.doi.org/10.4238/2011.October.5.3

Li SC, Yang L, Deng QM, Wang SQ, et al. (2006). Phenotypic characterization of a female sterile mutant in rice. J. Integr. Plant Biol. 48: 307-314. http://dx.doi.org/10.1111/j.1744-7909.2006.00228.x

Liu HZ, Zhang GS, Zhu WW, Ba QS, et al. (2015). Relationship between male sterility and $\beta$-1,3-glucanase activity and callose deposition-related gene expression in wheat (Triticum aestivum L.). Genet. Mol. Res. 14: 574-584. http:// dx.doi.org/10.4238/2015.January.26.12

Lorenzetti F (1993). Achieving potential herbage seed yield in species of temperate regions. In: Proceedings of the XVII International Grassland Congress. Palmerston North, 1621-1628.

Mariani A, Tavoletti S and Veronesi F (1993). Abnormal macrosporogenesis in five alfalfa (Medicago sativa) mutants producing $4 \mathrm{n}$ pollen. Theor. Appl. Genet. 85: 873-881.

Márton ML and Dresselhaus T (2008). A comparison of early molecular fertilization mechanisms in animals and flowering plants. Sex. Plant Reprod. 21: 37-52. http://dx.doi.org/10.1007/s00497-007-0062-8

Motamayor JC, Vezon D, Bajon C, Sauvanet A, et al. (2000). Switch (swi1), an Arabidopsis thaliana mutant affected in the female meiotic switch. Sex. Plant Reprod. 12: 209-218. http://dx.doi.org/10.1007/s004970050002

Musiał K, Kościńska-Pająk M, Antolec R and Joachimiak AJ (2015). Deposition of callose in young ovules of two Taraxacum species varying in the mode of reproduction. Protoplasma 252: 135-144. http://dx.doi.org/10.1007/ $\underline{\text { s00709-014-0654-8 }}$

Nair RM, Peck DM, Dundas IS, Samac DA, et al. (2008). Morphological characterization and genetic analysis of a bi-pistil mutant (bip) in Medicago truncatula Gaertn. Sex. Plant Reprod. 21: 133-141. http://dx.doi.org/10.1007/s00497-008-0073-0

Nishikawa S, Zinkl GM, Swanson RJ, Maruyama D, et al. (2005). Callose ( $\beta-1,3$ glucan) is essential for Arabidopsis pollen wall patterning, but not tube growth. BMC Plant Biol. 5: 22. http://dx.doi.org/10.1186/1471-2229-5-22

Okuda S, Tsutsui H, Shiina K, Sprunck S, et al. (2009). Defensin-like polypeptide LUREs are pollen tube attractants secreted from synergid cells. Nature 458: 357-361. http://dx.doi.org/10.1038/nature07882

Pagnussat GC, Yu HJ and Sundaresan V (2007). Cell-fate switch of synergid to egg cell in Arabidopsis eostre mutant embryo sacs arises from misexpression of the BEL1-like homeodomain gene BLH1. Plant Cell 19: 3578-3592. http:// 
dx.doi.org/10.1105/tpc. 107.054890

Palmer RG and Horner HT (2000). Genetics and cytology of a genic male-sterile, female-sterile mutant from a transposoncontaining soybean population. J. Hered. 91: 378-383. http://dx.doi.org/10.1093/jhered/91.5.378

Palmer RG, Sandhu D, Curran K and Bhattacharyya MK (2008). Molecular mapping of 36 soybean male-sterile, femalesterile mutants. Theor. Appl. Genet. 117: 711-719. http://dx.doi.org/10.1007/s00122-008-0812-5

Pasumarty SV, Matsumura T, Higuchi S and Yamada T (1993). Cultivar variation for seed development in white clover (Trifolium repens L.). Euphytica 65: 211-217. http://dx.doi.org/10.1007/BF00023085

Rosellini D, Lorenzetti F and Bingham ET (1998). Quantitative ovule sterility in Medicago sativa. Theor. Appl. Genet. 97: 1289-1295. http://dx.doi.org/10.1007/s001220051021

Rosellini D, Ferranti F, Barone P and Veronesi F (2003). Expression of female sterility in alfalfa (Medicago sativa L.). Sex. Plant Reprod. 15: 271-279.

Samuel MA, Chong YT, Haasen KE, Aldea-Brydges MG, et al. (2009). Cellular pathways regulating responses to compatible and self-incompatible pollen in Brassica and Arabidopsis stigmas intersect at Exo70A1, a putative component of the exocyst complex. Plant Cell 21: 2655-2671. http://dx.doi.org/10.1105/tpc.109.069740

Tao L, Yang Y, Wang Q and You X (2012). Callose deposition is required for somatic embryogenesis in plasmolyzed Eleutherococcus senticosus zygotic embryos. Int. J. Mol. Sci. 13: 14115-14126. http://dx.doi.org/10.3390/ ijms131114115

Teng N, Chen T, Jin B, Wu X, et al. (2006). Abnormalities in pistil development result in low seed set in Leymus chinensis (Poaceae). Flora 201: 658-667. http://dx.doi.org/10.1016/j.flora.2005.12.006

Vishnyakova MA (1991). Callose as an indicator of sterile ovules. Phytomorphology 41: 245-252.

Wang X, Liu H, Li X, Song Y, et al. (2009). Correlations between environmental factors and wild bee behavior on alfalfa (Medicago sativa) in northwestern China. Environ. Entomol. 38: 1480-1484. http://dx.doi.org/10.1603/022.038.0516

Wang XJ, Li XX, Zhang JW, Feng GH, et al. (2011). Characterization of nine alfalfa varieties for differences in ovule numbers and ovule sterility. Aust. J. Crop. Sci. 5: 447-452.

West M and Harada JJ (1993). Embryogenesis in higher plants: an overview. Plant Cell 5: 1361-1369. http://dx.doi. org/10.1105/tpc.5.10.1361

Winiarczyk K, Jaroszuk-Ściseł J and Kupisz K (2012). Characterization of callase ( $\beta$-1,3-D-glucanase) activity during microsporogenesis in the sterile anthers of Allium sativum L. and the fertile anthers of A. atropurpureum. Sex. Plant Reprod. 25: 123-131. http://dx.doi.org/10.1007/s00497-012-0184-5

Yang ZJ, Peng ZS, Yang H, Yang J, et al. (2011). Suppression subtractive hybridization identified differentially expressed genes in pistil mutations in wheat. Plant Mol. Biol. Rep. 29: 431-439. http://dx.doi.org/10.1007/s11105-010-0249-2

Zhang ZB, Zhu J, Gao JF, Wang C, et al. (2007). Transcription factor AtMYB103 is required for anther development by regulating tapetum development, callose dissolution and exine formation in Arabidopsis. Plant J. 52: 528-538. http:// dx.doi.org/10.1111/j.1365-313X.2007.03254.x 